\title{
Discordant Temporal Changes in Electrophysiological Properties during Electrical Remodeling and Its Recovery in the Canine Atrium
}

\author{
Akiko SHIROSHITA-TAKESHITA, ${ }^{1} \mathrm{MD}$, Hideo MitAmurA, ${ }^{1} \mathrm{MD}$, \\ Kaori ShInAgawA, ${ }^{1} \mathrm{MD}$, Toshiaki SATO,${ }^{1} \mathrm{MD}$, Yasuo KuRITA, ${ }^{1} \mathrm{MD}$, \\ Hideaki KANKI, ${ }^{1}$ MD, Seiji TAKATSUKI, ${ }^{1} \mathrm{MD}$, Motoki HaRA,${ }^{1} \mathrm{MD}$, \\ and Satoshi OGAWA, ${ }^{1} \mathrm{MD}$
}

\begin{abstract}
SUMMARY
Prolonged rapid atrial excitation gives rise to atrial electrical remodeling, which perpetuates atrial fibrillation (AF). However, there has been controversy regarding the nature of temporal changes in conduction characteristics during the development and recovery of electrical remodeling. This study was designed to clarify the nature of the development and recovery of electrical remodeling in relation to AF inducibility in dogs subjected to rapid atrial pacing. Eleven dogs underwent rapid atrial pacing (400/min) for 28 days. The electrophysiological study was performed on the day just prior to the commencement of pacing, on days $2,7,14$, and 28 of rapid pacing, as well as 1 and 7 days after the cessation of pacing. In response to rapid atrial pacing, atrial effective refractory period (ERP), conduction velocity and wavelength decreased significantly $(p<0.05)$. ERP had shortened significantly and rapidly within 2 days of pacing, while conduction velocity decreased more gradually. During the recovery, ERP returned to almost baseline levels within a day, whereas conduction velocity returned to baseline by day 7. Sustained AF became inducible in $37 \%$ of the dogs from 7 days of pacing until 1 day after the cessation, when wavelength fell below $8.7 \mathrm{~cm}$. In conclusion, rapid atrial excitation causes a progressive but discordant temporal pattern of a decrease in ERP and conduction velocity. The resultant shortening of the wavelength determines the inducibility and maintenance of AF. The electrophysiological changes produced by one month of rapid atrial pacing can be fully reversed within a week, although in a discordant temporal pattern. (Jpn Heart J 2002; 43: 167-181)
\end{abstract}

Key words: Atrial fibrillation, Electrophysiology, Rapid atrial pacing, Conduction characteristics, Reversibility

ATRIAL fibrillation (AF) is one of the most common arrhythmias encountered in clinical practice and is particularly prevalent among the older population. ${ }^{1-3)}$

From the ${ }^{1}$ Cardiopulmonary Division, Department of Medicine, Keio University School of Medicine, Tokyo, Japan.

Address for correspondence: Hideo Mitamura, MD, Cardiopulmonary Division, Department of Medicine, Keio University

School of Medicine, 35 Shinanomachi, Shinjuku-ku, Tokyo 160-8582, Japan.

Received for publication August 20, 2001.

Revised and accepted November 15, 2001. 
Sustained AF not only decreases cardiac output but also increases the risk of systemic thromboembolism with significant morbidity and mortality. In spite of general agreement concerning the importance in the management of $\mathrm{AF}$, the mechanisms of AF are not fully understood and the prevention of AF is still a challenge.

Clinical experience suggests that paroxysmal AF often progresses into persistent AF, and the longer AF persists, the more difficult it becomes to maintain sinus rhythm after cardioversion. An elegant experimental study by Wijffel, et al demonstrated that rapid atrial pacing-induced chronic AF in goats can cause electrical remodeling with atrial electrophysiological changes that are responsible for a phenomenon called "AF begets AF". ${ }^{4}$ ) Over the past few years, several studies have shown that rapid atrial excitation promotes shortening of the atrial effective refractory period (ERP) with a reduction in physiological ERP adaptation to rate, leading to an increase in the inducibility of $\mathrm{AF}^{5-7)}$

Recovery in atrial refractoriness from electrical remodeling is also important and can be related to the recurrence of AF after cardioversion. Several investigators have shown in animal models as well as in humans that these electrophysiological changes produced by either short or long-term rapid atrial excitation can be reversible. ${ }^{4,6,8-11)}$ However, the detailed time course of electrical remodeling and its recovery, especially with regard to conduction characteristics, are still controversial.

The purpose of this study was to clarify the detailed time course of electrical remodeling and its recovery by repeatedly measuring the changes in electrophysiological parameters and the inducibility of AF at various stages of chronic rapid atrial pacing, as well as during its recovery in dogs subjected to continuous rapid atrial pacing.

\section{Methods}

Chronic rapid atrial pacing model: Eleven mongrel dogs of either sex (mean weight, $14.0 \mathrm{~kg}$ ) were used in this study. Animal handling was approved by the Institutional Scientific Review Committee of Keio University and the investigation conformed with the guidelines specified by the Institutional Animal Care and Use Committee of Keio University School of Medicine. The dogs were anesthetized with sodium pentobarbital $(20 \mathrm{mg} / \mathrm{kg}$ IV) and respiration was maintained via an endotracheal tube and mechanical ventilation. A right intercostal thoracotomy was performed and the pericardium was opened to expose the heart. A custom-made silicon strip containing both a pair of bipolar pacing electrodes (interpolar distance $2 \mathrm{~mm}$ ) and two pairs of recording electrodes (interpolar distance $2 \mathrm{~mm}$, inter-electrode distance $15 \mathrm{~mm}$, Nihon Kohden, Japan) were sutured 
in alignment on the epicardium of the right atrial appendage. The arrangement of these electrodes is schematically illustrated in Figure 1. After closure of the pericardium and thorax, these electrode leads were tunneled subcutaneously to the back and then connected to a custom-made external pacemaker (Nihon Kohden) placed in a jacket. Antibiotics were given and the dogs were kept under careful observation during recovery. Five days after surgery, rapid atrial pacing at a rate of $400 / \mathrm{min}$ with $0.7-\mathrm{msec}$ pulses at 5 volts was started and continued for 28 days. Electrophysiological study: Programmable stimulators (cardiac stimulator, BC02A, Fukuda Denshi, Japan and pulse generator, 88-1346, Nihon Kohden) and isolators (SS-202J, Nihon Kohden) were used to deliver symmetric biphasic rectangular pulses with a 2-msec duration. A transducer (TP-400-T, Nihon Kohden), a direct-current coupled isolated amplifier with a frequency range of 0.04 to 500 $\mathrm{Hz}$, and a thermal array recorder (Polygraph system RM 6000, Nihon Kohden), were used to monitor and record surface electrocardiograms, atrial bipolar electrograms, and stimulus signals.

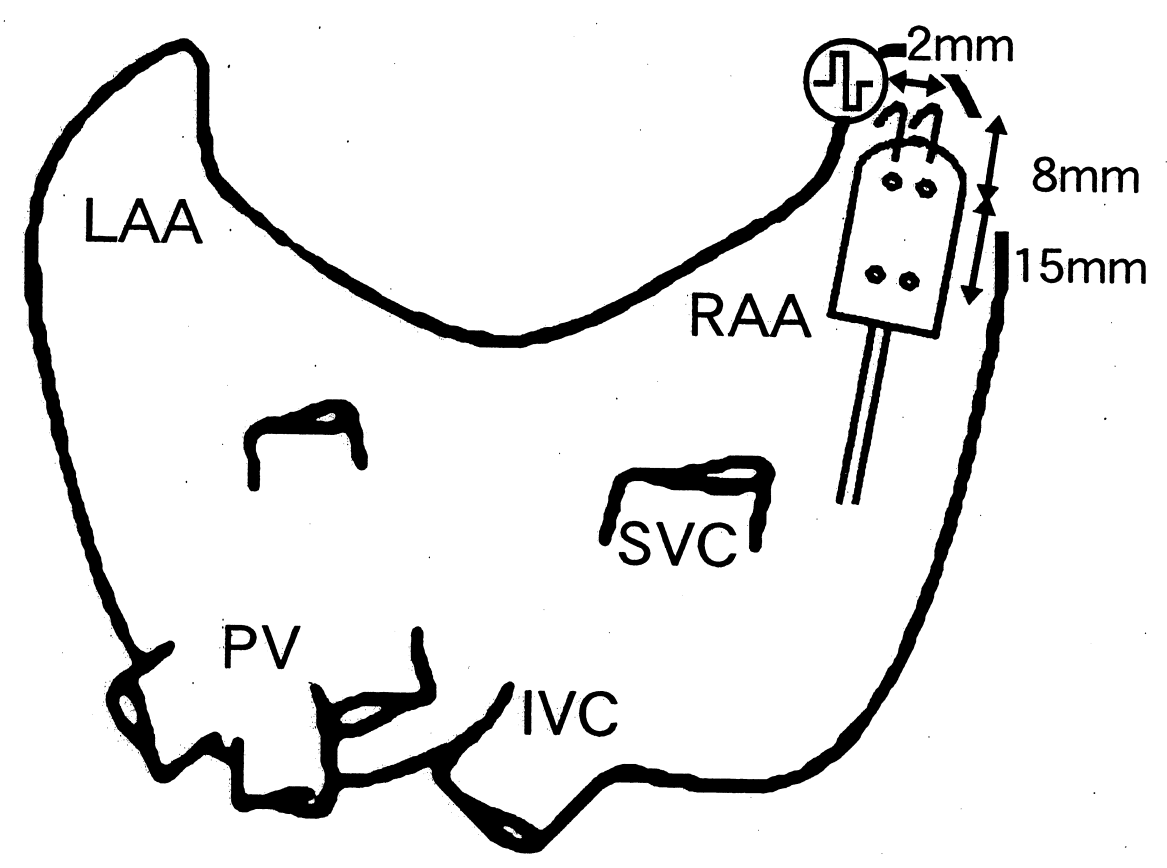

Figure 1. Arrangement of electrodes for pacing and recording. Custom-designed electrodes were sutured on the ridge of the right atrial appendage (RAA). A pair of electrodes at the distal hook served for pacing and 2 pairs of electrodes with an inter-electrode distance of $15 \mathrm{~mm}$ were aligned on a proximal sheet for recording. Each pair of electrodes had an interpolar distance of $2 \mathrm{~mm}$. SVC=superior vena cava; IVC=inferior vena cava; $\mathrm{PV}=$ pulmonic vein; LAA=left atrial appendage. 
All measurements were made on the day just prior to the commencement of rapid atrial pacing (P0), on day 2 (P2), day 7 (P7), day 14 (P14), and day 28 (P28) of continuous atrial pacing, as well as 1 day (PP1) and 7 days (PP7) after the cessation of pacing. The inducibility of AF was examined by burst atrial pacing using $50-\mathrm{Hz}$ pulses with a $2-\mathrm{msec}$ width at four times the diastolic threshold current. Sustained AF was defined as rapid and irregular atrial excitation with varying atrial electrogram morphology that was sustained for more than $15 \mathrm{sec}$. When $\mathrm{AF}$ was induced, the $\mathrm{AF}$ duration and the average fibrillation interval (FF interval) were measured.

Electrophysiological study was performed during sinus rhythm under pharmacological autonomic blockade using atropine $(0.04 \mathrm{mg} / \mathrm{kg} \mathrm{IV})$ and propranolol $(0.1 \mathrm{mg} / \mathrm{kg} \mathrm{IV})$. The atrial effective refractory period (ERP) was measured by a 2 msec-step decremental technique during constant pacing at cycle lengths (PCLs) of 400 and $200 \mathrm{msec}$ for 10 beats using four times the diastolic threshold current. ERP was defined as the longest S1-S2 coupling interval that failed to result in atrial capture.

The intra-atrial conduction velocity $(\mathrm{CV})$ was obtained by dividing the distance of $15 \mathrm{~mm}$ between the proximal pairs of recording electrodes by the conduction time for an impulse propagating between these electrodes during constant pacing at PCLs of 400 and $200 \mathrm{msec}$ from a pair of electrodes arranged $8 \mathrm{~mm}$ distal to the next proximal electrodes. To determine the conduction time, the time interval between the activation signals was obtained as measured at the onset of each electrogram defined by the first deflection from the baseline. The wavelength (WL) was calculated from the products of ERP and CV at PCLs of 400 and $200 \mathrm{msec}$.

Statistical analysis: Continuous variables are presented as the mean \pm SEM. Statistical comparisons of multiple group means were conducted using 2-way repeated ANOVA. A paired Student's $t$-test was used to compare the data where appropriate. The $\chi^{2}$ test with Fisher's exact test was used to assess nonparametric data. A value of $p<0.05$ was considered to be statistically significant.

\section{RESULTS}

Temporal changes in electrophysiological properties during rapid atrial pacing and their recovery after the cessation of pacing: In response to rapid atrial pacing, ERP shortened significantly accompanied by a reduction in its rate adaptation (Table I). Figure 2 shows the time course of ERP shortening during constant rapid atrial pacing over 28 days. ERP shortened significantly by P2, almost reaching a plateau by P7 at both PCLs. Compared to ERP shortening, CV decreased more gradually and progressively over 28 days of pacing (Figure 2). As a result of this 
Table I. Temporal Changes of Electrical Remodeling and Its Recovery

\begin{tabular}{|c|c|c|c|c|c|c|c|}
\hline & $\mathrm{P} 0$ & $\mathrm{P} 2$ & $\mathrm{P} 7$ & P14 & P28 & PP1 & PP7 \\
\hline ERP200 (msec) & $117 \pm 4$ & $111 \pm 3 *$ & $108 \pm 3 *$ & $105 \pm 2 *$ & $108 \pm 2 *$ & $114 \pm 2$ & $123 \pm 2$ \\
\hline ERP400 (msec) & $144 \pm 4$ & $127 \pm 3 *$ & $115 \pm 3 *$ & $117 \pm 4 *$ & $124 \pm 3 *$ & $139 \pm 4$ & $148 \pm 3$ \\
\hline $\mathrm{CV} 200(\mathrm{~cm} / \mathrm{sec})$ & $84.2 \pm 3.2$ & $78.7 \pm 3.3$ & $76.1 \pm 3.1 *$ & $71.8 \pm 2.4^{*}$ & $68.5 \pm 1.6^{*}$ & $73.8 \pm 2.5^{*}$ & $80.7 \pm 2.8$ \\
\hline WL200 $(\mathrm{cm})$ & $9.8 \pm 0.5$ & $8.7 \pm 0.3 *$ & $7.7 \pm 0.3 *$ & $7.6 \pm 0.3^{*}$ & $7.4 \pm 0.2 *$ & $8.5 \pm 0.3 *$ & $9.8 \pm 0.4$ \\
\hline AF induction (No. of dogs) & 0 & 0 & 4 & 5 & 6 & 4 & 0 \\
\hline AF duration (sec) & $0.5 \pm 0.3$ & $2.2 \pm 1.2$ & $45.4 \pm 26.7$ & $60.5 \pm 34.9$ & $101.1 \pm 40.1$ & $37.9 \pm 18.1$ & $2.1 \pm 1.1$ \\
\hline FF interval (msec) & $118 \pm 4$ & $116 \pm 5$ & $104 \pm 4$ & $93 \pm 3$ & $92 \pm 3$ & $118 \pm 6$ & $121 \pm 7$ \\
\hline
\end{tabular}

$\mathrm{P} 0=$ just prior to the commencement of rapid atrial pacing; P2, P7, P14 and P28=2, 7, 14, and 28 days after the onset of pacing; ERP200, ERP400=atrial effective refractory period obtained at pacing cycle length of 200 and 400 msec; CV200=intra-atrial conduction velocity obtained at pacing cycle length of $200 \mathrm{msec}$; WL200=wavelength obtained at cycle length of $200 \mathrm{msec}$; FF interval=average fibrillation interval. *: $p<0.05$ vs P0.

discordant time course of ERP shortening and CV decrease during rapid atrial pacing, WL shortened gradually over the whole pacing period of 28 days.

The recovery course of these electrophysiological changes in the remodeled atrium after the cessation of rapid pacing also displayed a discordant temporal pattern. The ERP increased rapidly, reaching approximately $97 \%$ of the baseline value together with regaining its normal rate adaptation by PP1 and had fully recovered by $\mathrm{PP} 7$. In contrast, $\mathrm{CV}$ returned more slowly, taking 7 days after the cessation of rapid atrial pacing to reach the baseline level (Figure 3). As a result of this discordant temporal pattern of recovery for ERP and CV, WL gradually returned to the baseline by PP7. Of note was the fact that the changes in electrophysiological parameters produced by 28 days of rapid atrial excitation were almost fully reversed within 7 days of sinus rhythm after the cessation of pacing. Temporal changes in AF inducibility and AF duration in relation to electrical remodeling and its recovery: Figure 4 shows an example of the temporal changes in AF induction during rapid atrial pacing and after the cessation of pacing. On P0 and P2, only non-sustained AF was induced, and the mean FF interval was relatively long (135 msec and $122 \mathrm{msec}$, respectively). Sustained AF became inducible on P7 and thereafter, and its duration became progressively longer during the entire pacing period. The mean FF interval progressively shortened as the duration of pacing lengthened (97 msec on P7 to $83 \mathrm{msec}$ on P28). After the cessation of pacing, the mean FF interval became prolonged again to $110 \mathrm{msec}$ on PP1, and sustained AF was no longer inducible by PP7. The temporal changes in AF inducibility, AF duration, and the mean FF interval were similar in all of the dogs studied (Table I).

In summary, sustained AF was not induced in any dog on P0 and P2, however, sustained AF became inducible in 4, 5, and 6 cumulative dogs out of the 11 dogs on P7, P14, and P28, respectively. Sustained AF remained still inducible in 

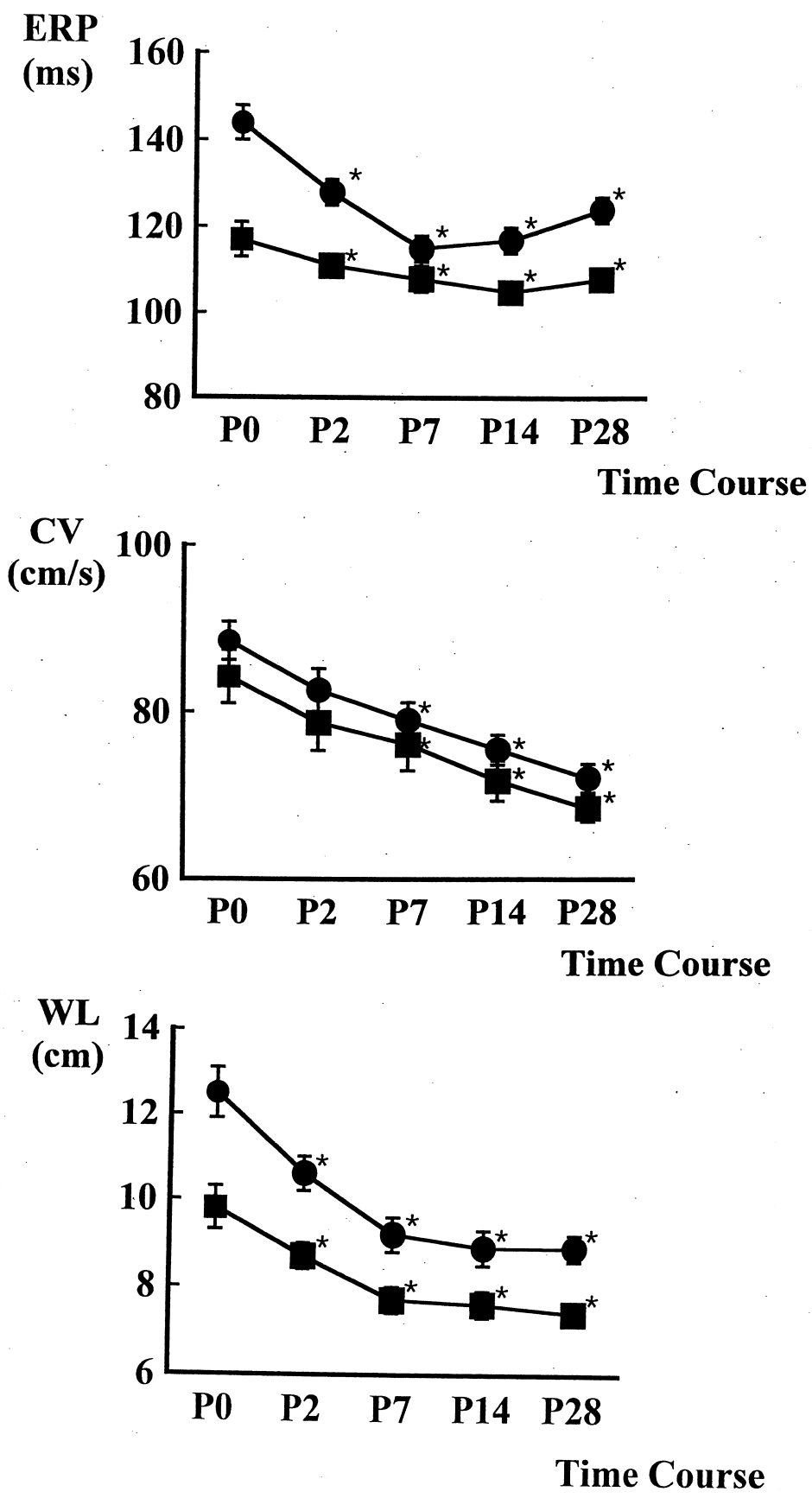

Figure 2. Temporal changes in ERP (top), CV (middle), and WL (bottom) at PCL of 200 (square) and $400 \mathrm{msec}$ (circle) during rapid atrial pacing. ERP shortened significantly with reduction of rate adaptation by P2 and reached a steady state level by P7. In contrast to ERP shortening, CV decreased more gradually throughout the whole pacing period. As a result of ERP shortening and CV decrease, WL also decreased gradually and fell below $8.7 \mathrm{~cm}$ at PCL of $200 \mathrm{msec}$ after P7. *: $p<0.05$ vs P0. 

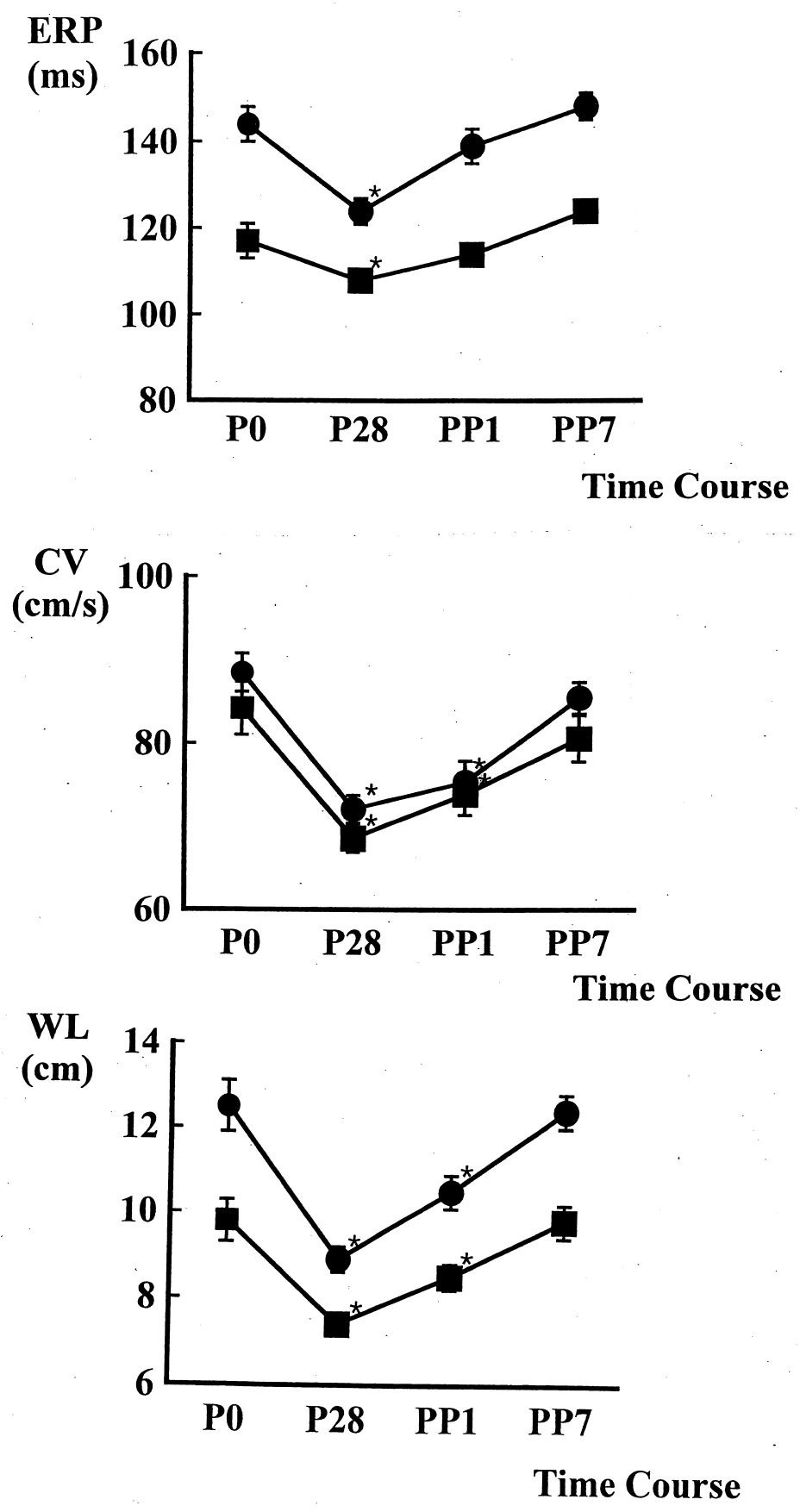

Figure 3. Temporal changes in ERP (top), CV (middle), and WL (bottom) at PCL of 200 (square) and $400 \mathrm{msec}$ (circle) during the recovery course after the cessation of rapid atrial pacing. ERP returned to $97 \%$ of the baseline value just 1 day after the cessation of rapid atrial pacing (PP1), whereas CV returned more slowly to the baseline value 7 days after the cessation of pacing (PP7). WL also returned with a slower course after the cessation of pacing. *: $p<0.05$ vs $\mathrm{P} 0$. 


\section{AF Induction}

50-Hz burst

P0
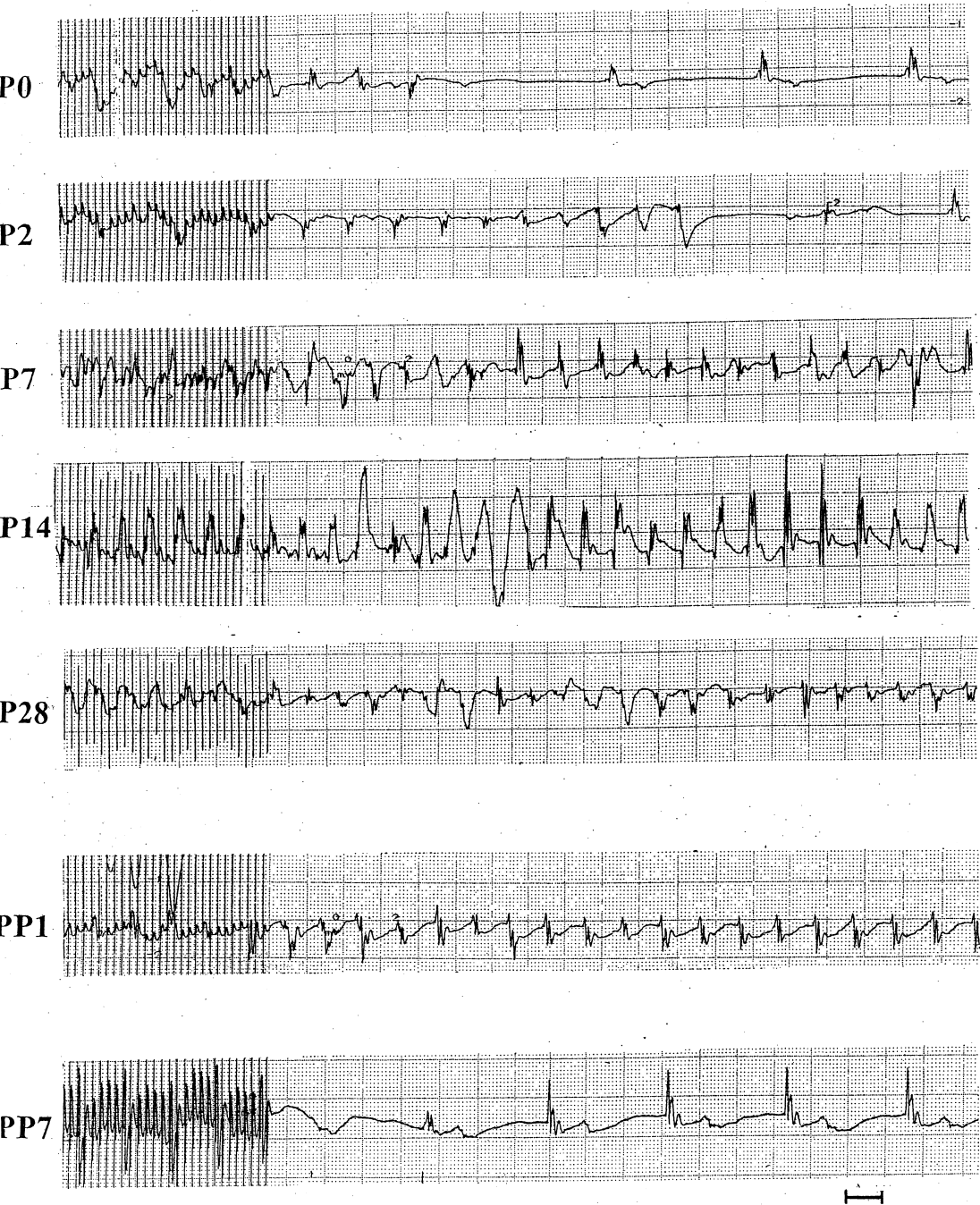

$100 \mathrm{msec}$

Figure 4. Typical example of the temporal changes in AF inducibility both during rapid atrial pacing and post-pacing period. Traces of bipolar electrograms when the inducibility of AF was examined by burst atrial pacing using currents of $50 \mathrm{~Hz}$ on each of the study days are shown. The mean fibrillation interval (FF interval) is shown on the right. Sustained AF was not inducible on either P0 or P2, and the FF intervals of non-sustained AF were relatively long (135 msec and 122 msec, respectively). Sustained AF became inducible after P7, AF duration became longer, and FF interval progressively shortened as the duration of rapid pacing increased (97 msec on P7 to $83 \mathrm{msec}$ on P28). In the recovery course, AF was still inducible one day after the cessation of pacing (PP1), but was no longer inducible by PP7. 
4 of the 11 dogs on PP1, but was no longer inducible by PP7 (Figure 5). The duration of $\mathrm{AF}$ increased as the duration of rapid pacing was prolonged. The average duration of induced AF was prolonged progressively from $0.5 \mathrm{sec}$ on $\mathrm{P} 0$ to 45.4

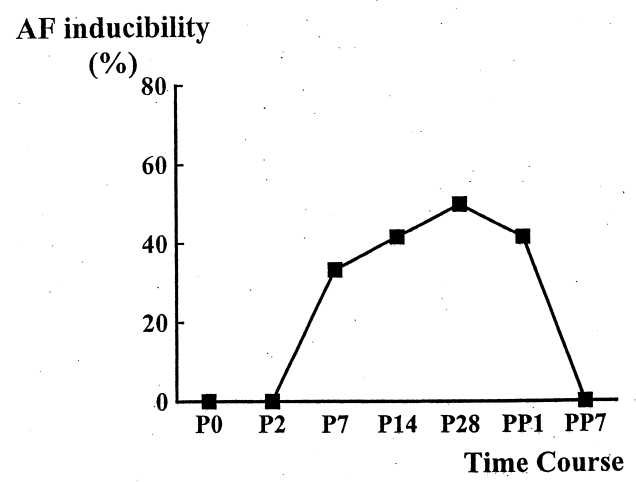

AF duration

(sec)

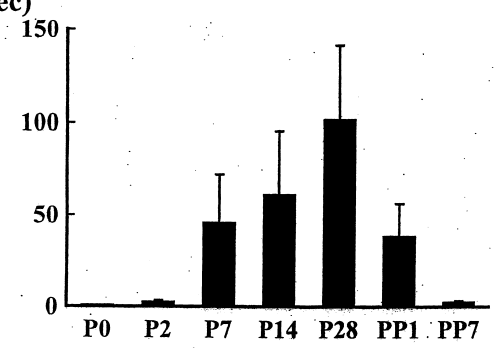

Time Course

FF interval

(msec)

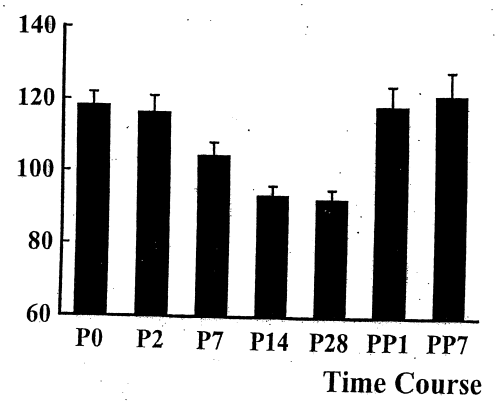

Figure 5. Temporal changes of AF inducibility (top), duration of $\mathrm{AF}$ (middle), and mean FF interval (bottom) during electrical remodeling and its recovery. Sustained AF was not induced in any dog on $\mathrm{P} 0$ and $\mathrm{P} 2$; however, it was inducible in 4, 5, and 6 cumulative dogs out of the 11 dogs on P7, P14, and P28, respectively. AF remained inducible in 4 of the 11 dogs on PP1, but was no longer inducible by PP7. The duration of AF became longer as the duration of rapid pacing was increased. The duration of induced AF was prolonged progressively from $0.5 \mathrm{sec}$ on P0 to $45.4 \mathrm{sec}$ on P7 and $101.1 \mathrm{sec}$ on P28. After the cessation of pacing, it shortened rapidly to $37.9 \mathrm{sec}$ on PP1 and $2.1 \mathrm{sec}$ on PP7. Moreover, the FF interval shortened progressively from 118 msec on P0 to $104 \mathrm{msec}$ on P7 and $92 \mathrm{msec}$ on P28, and progressively recovered by PP1 to $118 \mathrm{msec}$. 
sec on P7 and $101.1 \mathrm{sec}$ on P28. After the cessation of pacing, it shortened rapidly to $37.9 \mathrm{sec}$ on PP1 and $2.1 \mathrm{sec}$ on PP7 (Figure 5). Moreover, the FF interval shortened progressively from $118 \mathrm{msec}$ on P0 to $104 \mathrm{msec}$ on P7 and $92 \mathrm{msec}$ on P28, and progressively recovered to $118 \mathrm{msec}$ by PP1 (Figure 5). There was a significant relationship between AF inducibility and WL $(p=0.0001)$ such that sustained AF was inducible exclusively when WL obtained at PCL $200 \mathrm{msec}$ was shorter than $8.7 \mathrm{~cm}$ (Figure 6), which corresponded to a duration of rapid atrial pacing for 7 days or longer and was observed until 1 day after the cessation of pacing.

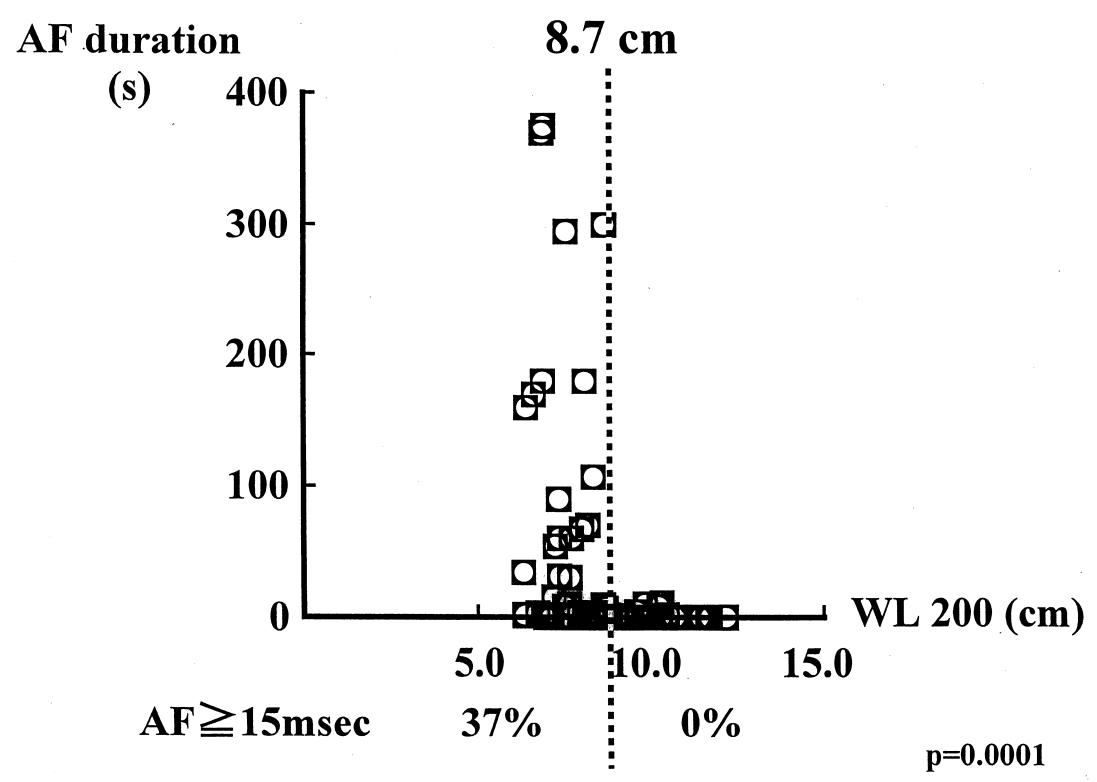

Figure 6. Relation between AF inducibility and WL obtained at a PCL of $200 \mathrm{msec}$. Sustained AF was induced in $37 \%$ of the dogs when WL fell below $8.7 \mathrm{~cm} . p=0.0001$.

\section{DisCUSSION}

Main findings: In the present study, the time course of atrial electrical remodeling and its recovery have been evaluated by serially measuring electrophysiological parameters and by attempting the induction of AF in dogs subjected to one month of continuous rapid atrial pacing. The results suggest that rapid atrial excitation causes a progressive shortening of ERP and a decrease of $\mathrm{CV}$, leading to a shortening of WL along with a progressive increase in AF inducibility and its duration. In this study, the temporal changes in ERP and CV during electrical remodeling displayed a discordant pattern, where the ERP shortening progressed 
faster and was almost completed in 7 days, while CV and WL decreased more gradually and progressively over the 28 days of rapid atrial pacing. Moreover, the recovery from electrical remodeling after the cessation of pacing also took a discordant temporal course. ERP reached the baseline value within a day after the termination of pacing, while CV and WL returned to baseline levels more slowly over 7 days with a decrease in AF inducibility and its duration.

Our results also demonstrated that the inducibility of AF was related to the degree of electrical remodeling and was determined by the value of WL. Sustained AF became inducible after 7 days of rapid atrial pacing and remained inducible until 1 day after the cessation of pacing, which corresponded to a WL shorter than $8.7 \mathrm{~cm}$. Surprisingly, all of the electrophysiological changes including AF inducibility, its duration, and FF interval brought about by one month of rapid atrial excitation were fully reversed within 7 days of the post-pacing period.

Electrophysiological changes due to rapid atrial activation: Morillo, et $a l^{5)}$ first reported in a canine model that ERP shortened and AF inducibility increased after 6 weeks of rapid atrial pacing. Wijffels, et $a l^{4)}$ demonstrated in an instrumented AF goat model that sustained AF led to a shortening of atrial refractoriness and its loss of physiological adaptation to rate. Gaspo, et $a l^{7)}$ also demonstrated in dogs undergoing rapid atrial pacing of various duration that ERP shortening and $\mathrm{CV}$ decrease took place in a progressive and discordant manner in accordance with our results. Unlike our study, however, they examined 3 groups of dogs subjected to rapid atrial pacing for 1, 7, and 42 days that were compared with a group of sham-operated dogs. ${ }^{7}$ Therefore, information about detailed temporal changes during the subacute phase of electrical remodeling such as for days 2, 14, and 28 was not obtained in their study, which is also important and clinically relevant if one considers whether the elective cardioversion of AF should take place within 2 days or after one month of anticoagulation. Moreover, as they compared different groups of animals, their results had the drawback of including inter-group differences. The present study was designed to serially measure electrophysiological parameters in the same dogs to make paired comparisons possible and to provide more precise data regarding the time course of electrical remodeling and its recovery at different stages ranging from the baseline, 2 days, 7 days, 14 days, and 28 days of rapid pacing, as well as 1 and 7 days of sinus rhythm after the cessation of pacing.

Reversibility of pacing-induced electrical remodeling: The present study has demonstrated a complete reversibility of electrophysiological changes from a decrease in ERP, CV, and WL and an increase in the inducibility of AF resulting from rapid atrial pacing over a one month period. Wijffels, et $a l^{4)}$ reported that after defibrillation of AF in 5 goats, ERP returned to its baseline value and began 
to exhibit normal rate adaptation after one week of sinus rhythm. There have been several other reports also showing that shortened ERP after sustained atrial activation was primarily prolonged significantly within a day or a few days of maintained sinus rhythm and returned to baseline within a week both in animal models $\mathrm{s}^{6,9,11)}$ and in humans ${ }^{10)}$ in accordance with our results.

However, there have been only a few reports describing the change in intraatrial conduction after termination of rapid atrial excitation. $\mathrm{Yu}$, et al ${ }^{10)}$ reported that there was no significant change either in intra-atrial conduction time or Pwave duration during an observation period of 4 days after electrical cardioversion in patients who had chronic AF for longer than 6 months. This suggests that long-term atrial fibrillation may induce far advanced remodeling, which would make conduction slowing difficult to reverse. Moriguchi, et al ${ }^{12)}$ examined atrial conduction time in dogs subjected to rapid atrial pacing for 14 days. They reported that the conduction time gradually prolonged during the 14 days of rapid pacing and shortened slowly over 7 days in the recovery phase. Their data may not be applicable to clinical practice where AF is usually terminated either within 2 days or after more than 3 weeks of anticoagulation.

In this regard, the present study is important where we measured CV repeatedly in the same dogs at various stages over one month of electrical remodeling and its recovery for 7 days. The decreases in CV and WL produced by one month of rapid atrial pacing were fully reversible within a week after the termination of pacing, supporting the hypothesis that one month of electrical remodeling may not yet lead to irreversible remodeling, allowing complete recovery of electrophysiological properties.

Mechanisms of discordant temporal changes in ERP and CV: The exact mechanism responsible for electrical remodeling and its recovery has not yet been fully elucidated, but some relevant findings have been reported. Pathological findings of the atria subjected to prolonged rapid excitation ${ }^{5,8,13)}$ and the efficacy of verapamil in preventing electrical remodeling, ${ }^{8,14-16)}$ both indirectly support the hypothesis that electrical remodeling may at least, in part, be related to cytosolic $\mathrm{Ca}$ overload. Ausma, et al recently reported that $\mathrm{Ca}^{2+}$ deposits along the sarcolemma and in the mitochondria increased markedly at 1 and 2 weeks after the initiation of AF in the goat model, ${ }^{17)}$ also indicating cellular Ca overload associated with sustained AF.

Using a voltage-clamp technique, Yue, et $a l^{18)}$ found a significant decrease in the membrane L-type Ca current of canine atria after 1 day of rapid atrial pacing, which was temporally concordant with shortening of the action potential duration. Gaspo, et $a l^{19)}$ also reported a decrease in the membrane Na current in the same chronic dog model. Their data show that Ca density was reduced by 52 $\%$, in contrast to only a $28 \%$ reduction in $\mathrm{Na}$ density of the canine atria paced for 
7 days. This difference in the degree of downregulation of membrane ionic currents may explain our findings of discordant pattern of temporal changes in electrophysiological parameters where ERP shortened faster, but CV decreased more slowly. Furthermore, van der Velden, et al reported on the time course of gap junctional remodeling in the goat model. ${ }^{20)}$ They showed that whereas the connexin 40 distribution in the atria was almost homogenous within 1 week of AF, it became heterogeneous with increasing duration of AF. Therefore, the discordant temporal changes in ERP and CV produced by rapid atrial excitation may be reflecting these multiple discordant temporal changes in the membrane L-type $\mathrm{Ca}$ current and $\mathrm{Na}$ current, as well as gap junctional protein.

This study is the first to describe the full reversibility of intra-atrial conduction delay that developed over one month of rapid atrial excitation. Although the reversibility of changes in membrane ionic currents or in the mRNA concentration of ionic channels in the remodeled atrium was not evaluated in this study, our results demonstrated that there was almost complete reversibility in the changes in the electrophysiological parameters and AF inducibility in the canine atrium subjected to rapid atrial pacing for as long as one month.

Temporal changes in AF inducibility in relation to wavelength: Atrial size and atrial wavelength have been considered important factors responsible for the perpetuation of $\mathrm{AF}^{21-23)}$ Gaspo, et $a l^{4)}$ reported that sustained AF could become inducible in dogs subjected to prolonged rapid atrial pacing. They showed that sustained AF was inducible in 54\% of dogs with 7 days of rapid pacing and in $86 \%$ of those with 42 days of pacing. Sustained AF became more frequently inducible and the mean AF cycle length shortened as the duration of pacing increased. They also showed that AF duration was strongly correlated with WL. The results of the present study are in accordance with their results. Characteristically, sustained AF became inducible after 7 days of rapid atrial pacing until one day after the cessation of pacing, when WL at a PCL of $200 \mathrm{msec}$ fell below 8.7 $\mathrm{cm}$ in this study.

Tieleman, et $\mathrm{l}^{14)}$ reported that AF tends to recur early after cardioversion of sustained AF in humans. They found a high incidence of AF relapse during the first 5 days after cardioversion. In the present study, AF was still inducible 1 day after the cessation of pacing when WL fell below $8.7 \mathrm{~cm}$, but was no longer inducible one week later when WL increased above this value. The present study indicates that $\mathrm{AF}$ inducibility depends not only on the duration of rapid atrial activation, but also on the duration of sinus rhythm after its resumption, both of which are critically determined by WL.

Limitations of the study: Electrophysiological variables were measured only at a single site on the right atrial appendage in this study and were not examined at other atrial sites. Thus, our study did not cover the dispersion of electrophysiolog- 
ical parameters in the whole atria. As Fareh, et al have noted ${ }^{24)}$ heterogeneity of atrial refractoriness has the potential to play an important role in the perpetuation of AF.

Another limitation of this study is the duration of rapid atrial pacing. This study was designed to characterize electrical remodeling created by 28 days of rapid atrial pacing at a rate of $400 / \mathrm{min}$. It is possible that the duration was not long enough to create advanced structural remodeling, which can develop later than electrical remodeling and may also affect arrhythmogeneity. Thus, the findings in this study may not be extrapolated to clinical practice where atrial tachyarrhythmias are sometimes sustained for months or even years.

Clinical implications: The present study has revealed that electrical remodeling produced within 48 hours of rapid atrial pacing did not cause significant changes in conduction and wavelength. The fact that AF inducibility still remained low at 48 hours of electrical remodeling supports the belief that early cardioversion may be beneficial in clinical practice. However, an ACCP guideline ${ }^{25)}$ advises that conversion of persistent AF sustained for longer than 48 hours be postponed for at least 3 weeks of full anticoagulation to prevent embolic events. The data obtained at 28 days of electrical remodeling is also clinically relevant in this regard. At this stage, with ERP, CV and WL all decreasing, termination of AF would be more difficult.

The current study also suggests that the inducibility of AF can still be high one day after conversion but decreases significantly after one week. Although data is not given on the reversibility of atrial contraction and associated risk of thromboembolism, this study suggests that if it is possible to limit the whole duration of AF to within one month, the risk of recurrence after cardioversion will be significantly reduced following successful management of maintaining sinus rhythm for one week after defibrillation. This study thus provides a new and important basis for formulating strategy in the management of AF.

\section{REFERENCES}

1. Kannel WB, Wolf PA. Epidemiology of atrial fibrillation. In: Falk RH, Podrid PJ, editors. Atrial Fibrillation: Mechanisms and Management. New York: Raven Press, 1992: 82-92.

2. The National Heart, Lung, and Blood Institute Working Group on Atrial Fibrillation. Atrial Fibrillation: Current understandings and research imperatives. J Am Coll Cardiol 1993; 22: 1830-4.

3. Pritchett ELC. Management of atrial fibrillation. N Eng J Med 1992; 326: 1264-71.

4. Wijffels MC, Kirchhof CJ, Dorland R, Allessie MA. Atrial fibrillation begets atrial fibrillation. A study in awake chronically instrumented goats. Circulation 1995; 92: 1954-68.

5. Morillo CA, Klein GJ, Jones DL, Guiraudon CM. Chronic rapid atrial pacing. Structural, functional, and electrophysiological characteristics of a new model of sustained atrial fibrillation. Circulation 1995; 91: 1588-95. 
6. Elvan A, Wylie K, Zipes DP. Pacing-induced chronic atrial fibrillation impairs sinus node function in dogs. Electrophysiological remodeling. Circulation 1996; 94: 2953-60.

7. Gaspo R, Bosch RF, Talajic M, Nattel S. Functional mechanisms underlying tachycardia-induced sustained atrial fibrillation in a chronic dog model. Circulation 1997; 96: 4027-35.

8. Goette A, Honeycut C, Langberg JL. Electrical remodeling in atrial fibrillation. Time course and mechanisms. Circulation 1996; 94: 2968-74.

9. Lee SH, Lin FY, Yu WC, et al. Regional differences in the recovery course of tachycardia-induced changes of atrial electrophysiological properties. Circulation 1999; 99: 1255-64.

10. Yu WC, Lee SH, Tai CT, et al. Reversal of atrial electrical remodeling following cardioversion of long-standing atrial fibrillation in man. Cardiovasc Res 1999; 42: 470-6.

11. Wijffels MCEF, Kirchhof CJHJ, Dorland R, Power J, Allessie MA. Electrical remodeling due to atrial fibrillation in chronically instrumented conscious goats: Roles of neurohumoral changes, ischemia, atrial stretch, and high rate of electrical activation. Circulation 1997; 96: 3710-20.

12. Moriguchi M, Niwano S, Yoshizawa N, et al. Inhomogeneity in the appearance of electrical remodeling during chronic rapid atrial pacing. Evaluation of the dispersion of atrial effective refractoriness. Jpn J Circ 2001; 65: 335-40.

13. Ausma J, Wijffels M, Thone F, Wouters L, Allessie M, Borgers M. Structural changes of atrial myocardium due to sustained atrial fibrillation in the goat. Circulation 1997; 96: 3157-63.

14. Tieleman RG, Van Gelder IC, Crijns HJGM, et al. Early recurrences of atrial fibrillation after electrical cardioversion: a result of fibrillation-induced electrical remodeling of the atria? J Am Coll Cardiol 1998; 31: 167-73.

15. Tieleman RG, De Langen CDJ, Van Gelder IC, et al. Verapamil reduces tachycardia-induced electrical remodeling of the atria. Circulation 1997; 95: 1945-53.

16. Daoud EG, Knight BP, Weiss R, et al. Effect of verapamil and procainamide on atrial fibrillation-induced electrical remodeling of the atria. Circulation 1997; 96: 1542-50.

17. Ausma J, Dispersyn GD, Duimel H, et al. Changes in ultrastructural calcium distribution in goat atria during atrial fibrillation. J Moll Cell Cardiol 2000; 32: 355-64.

18. Yue L, Feng J, Gaspo R, Li GR, Wang Z, Nattel S. Ionic remodeling underlying action potential changes in a canine model of atrial fibrillation. Circ Res 1997; 81: 512-25.

19. Gaspo R, Bosch RF, Abboud EB, Nattel S. Tachycardia-induced changes in $\mathrm{Na}^{+}$current in a chronic dog model of atrial fibrillation. Circ Res 1997; 81: 1045-52.

20. Van der Velden HMW, Ausma J, Rook MB, et al. Gap junctional remodeling in relation to stabilization of atrial fibrillation in the goat. Cardiovasc Res 2000; 46: 476-86.

21. Moe GK, Abildskov JA. Atrial fibrillation as a self-sustaining arrhythmia independent of focal discharge. Am Heart J 1959; 58: 59-70.

22. Moe GK. On the multiple wavelet hypothesis of atrial fibrillation. Arch Int Pharmacodyn 1962; 140: 183-8.

23. Moe GK, Rheinboldt WC, Abildskov JA. A computer model of atrial fibrillation. Am Heart J 1964; 67: 20020.

24. Fareh S, Villemaire C, Nattel S. Importance of refractoriness heterogeneity in the enhanced vulnerability to atrial fibrillation induction caused by tachycardia-induced atrial electrical remodeling. Circulation 1998; 98 : 2202-9.

25. American College of Chest Physicians. Third American College of Chest Physicians' Consensus Conference on Antithrombotic Therapy. Chest 1992; 102 (suppl 4) : 303S-549S. 